Musées, Patrimoine et Culture scientifiques et techniques

142 | 2012

juillet-août 2012

\title{
Évaluation normalisée de l'environnement du patrimoine
}

\section{Michel Dubus et Tomas Prosek}

\section{OpenEdition \\ Journals}

Édition électronique

URL : http://journals.openedition.org/ocim/1082

DOI : $10.4000 /$ ocim. 1082

ISSN : 2108-646X

Éditeur

OCIM

Édition imprimée

Date de publication : 1 juillet 2012

Pagination : $15-20$

ISSN : 0994-1908

Référence électronique

Michel Dubus et Tomas Prosek, «Évaluation normalisée de l'environnement du patrimoine », La Lettre de I'OCIM [En ligne], 142 | 2012, mis en ligne le 01 juillet 2014, consulté le 19 avril 2019. URL : http:// journals.openedition.org/ocim/1082 ; DOI : 10.4000/ocim.1082 


\section{Évaluation normalisée de l'environnement du patrimoine}

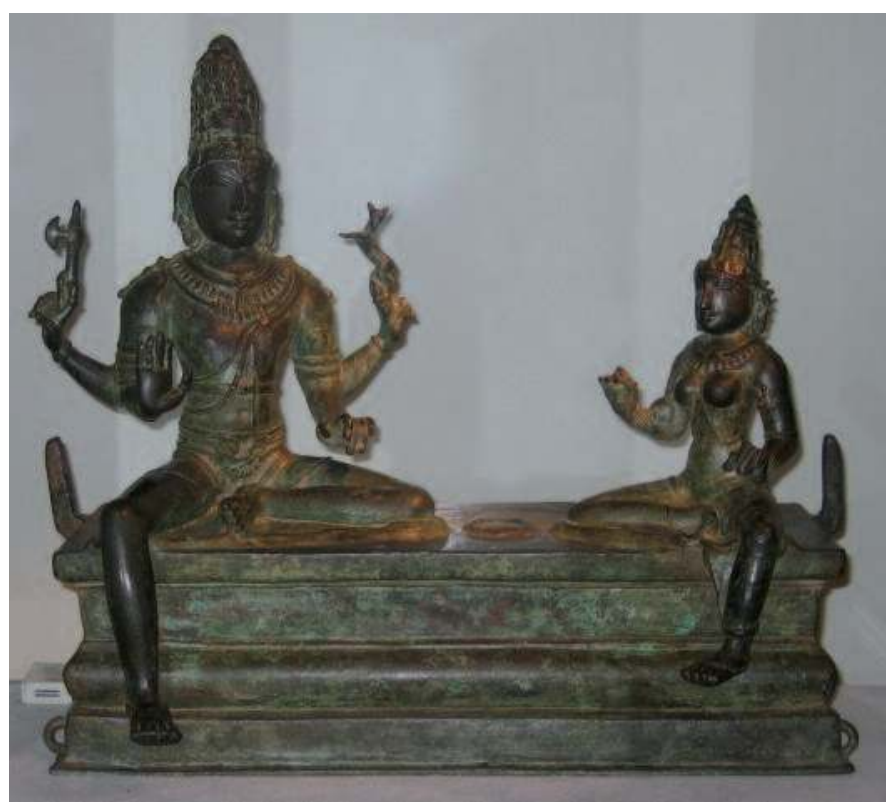

La vitrine Inde du musée Guimet. Les capteurs destinés à mesurer la corrosivité sont visibles en bas à gauche de la sculpture.

*Michel Dubus est ingénieur d'études au département Conservation préventive du Centre de Recherche et de Restauration des Musées de France michel.dubus@culture.gouv.fr Tomas Prosek est chercheur à l'Institut de la Corrosion SAS (ICO), Brest tomas.prosek@institut-corrosion.fr

\section{Michel Dubus et Tomas Prosek *}

La méthode de mesurage de la corrosion du cuivre décrite ici a été utilisée pour évaluer les conditions de conservation des collections patrimoniales : les résultats d'études réalisées dans des conditions de conservation ou d'exposition permanentes ou temporaires montrent que cette méthode est un outil efficace qui permet non seulement de comparer les bâtiments, les vitrines, les réserves mais aussi de les classer en fonction de leur corrosivité.

\footnotetext{
«Alors je me commençay à tormenter, pour savoir qui estoit la cause de cela, et ne pouvant entendre par Théorique, que Philosophie naturelle, il me prit désir de l'entendre par Pratique... » Bernard Palissy, Recepte véritable. Genève: Droz, 1988, p. 107.
}

\section{Introduction}

Dans les musées, les bibliothèques et les archives, les gaz ou particules en suspension dans l'air provenant de l'extérieur, des matériaux de construction, voire des collections elles-mêmes, peuvent altérer les matériaux, plus ou moins vite selon leur concentration : le dioxyde de soufre $\left(\mathrm{SO}_{2}\right)$ corrode les métaux, les alliages, les minéraux, attaque la cellulose, les colorants, les pigments et les photographies, le cuir ; le dioxyde d'azote $\left(\mathrm{NO}_{2}\right)$ favorise l'action du $\mathrm{SO}_{2}$ (le rôle de l'humidité étant moins important dans l'air intérieur qu'en milieux industriel et urbain) ; le sulfure d'hydrogène $\left(\mathrm{H}_{2} \mathrm{~S}\right)$ noircit l'argent, ternit le cuivre 
et ses alliages, certains pigments et colorants ; les acides organiques corrodent les aciers, les alliages cuivreux, le plomb, le zinc, le nickel, le carbonate de calcium ${ }^{(1)}$.

La croissance de films de corrosion sur des coupons de cuivre ou d'argent donne une excellente indication du type et de la concentration de ces polluants. L'industrie a développé des techniques basées sur ces propriétés pour obtenir sur le terrain des données expérimentales et rédigé des normes pour les interpréter. La recherche sur la qualité de l'air intérieur s'est d'abord intéressée aux standards téléphoniques dans les années 1930, puis dans les années 1960 aux centres de calcul, enfin au patrimoine à partir des années 1970 (2). En France, la qualité de l'air intérieur des logements n'est étudiée que depuis dix ans ${ }^{(3)}$, les archives, bibliothèques et musées restant terra incognita. Une étude a donc été menée pour évaluer les conditions de conservation dans plus de 70 environnements patrimoniaux, des musées d'ethnographie, des musées scientifiques et techniques, des archives et des bibliothèques pour les deux tiers.

Après avoir testé plusieurs méthodes basées sur la mesure du gain de masse des produits de corrosion telles que la microbalance à cristal à quartz ou la réduction coulométrique, ou encore des méthodes optiques ou électriques (4), nous avons choisi de mesurer la résistance d'un circuit électrique pour évaluer les films de corrosion formés. Pour estimer la qualité globale des atmosphères d'intérieur à partir de leur capacité à provoquer une corrosion, nous avons utilisé la norme internationale ISO 11844-1 qui a pour objet « de caractériser les environnements atmosphériques d'intérieur à faible corrosivité qui peuvent affecter les métaux et les revêtements métalliques au cours du stockage, du transport, de l'installation et de l'utilisation; d'établir une méthode cohérente de classification de la corrosivité de ces atmosphères; de spécifier des procédures de détermination et d'appréciation des catégories de corrosivité des atmosphères d'intérieur ». Cette norme est particulièrement bien adaptée car elle comprend une annexe spécifique permettant de déterminer la vitesse de corrosion par mesurage de la résistance sur une durée d'un an ${ }^{(5)}$.

\begin{tabular}{|lccccc|}
\hline Corrosivité & $\begin{array}{c}\text { IC } 1 \\
\text { très faible }\end{array}$ & $\begin{array}{c}\text { IC } 2 \\
\text { faible }\end{array}$ & $\begin{array}{c}\text { IC } 3 \\
\text { moyenne }\end{array}$ & $\begin{array}{c}\text { IC } 4 \\
\text { haute }\end{array}$ & $\begin{array}{c}\text { IC } 5 \\
\text { très haute }\end{array}$ \\
$\mathbf{n m} /$ mois & $\leq 5,6$ & $\leq 22$ & $\leq 101$ & $\leq 224$ & $\leq 561$ \\
\hline
\end{tabular}

Classification de la corrosivité du cuivre en atmosphères intérieures pour une durée d'exposition d'un an d'après ISO $11844-1$
Dans la norme, chaque classe de corrosion est divisée en deux sous ensembles décrivant les environnements typiques d'espaces chauffés ou non chauffés :

- IC 1 : corrosivité très faible correspondant à des «Espaces chauffés à humidité relative stable contrôlée $(<40 \%)$ sans risque de condensation, faibles niveaux de polluants, pas de polluants spécifiques... par exemple musées à environnements contrôlés» ou à des « Espaces non chauffés avec déshumidification, faibles degrés de pollution d'intérieur, pas de polluants spécifiques, par exemple dépôts de fournitures militaires».

- IC2 : corrosivité faible correspondant à des «Espaces chauffés à humidité relative faible $(<50 \%)$ avec une certaine variation d'humidité relative sans risque de condensation, faibles degrés de pollution, sans polluants spécifiques, par exemple musées, salles de commandes " ou à des "Espaces non chauffés avec uniquement des variations de température et d'humidité, sans risque de condensation, faibles degrés de pollution sans polluants spécifiques, par exemple salles de stockage avec faible fréquence de variations de température».

- IC3 : corrosivité moyenne, correspondant à des «Espaces chauffés avec risque de variation de température et d'humidité, degrés moyens de pollution, certain risque de présence de polluants spécifique, par exemple tableau de distribution dans l'industrie de l'énergie" ou à des "Espaces non chauffés à humidité relative élevée $(<50 \%-70 \%)$ avec variation périodique de l'humidité relative, sans risque de condensation, degrés élevés de pollution, faible risque de présence de polluants spécifiques, par exemple églises dans des zones non polluées, cabines téléphoniques extérieures dans des zones rurales ».

- IC4 : corrosivité forte correspondant à des «Espaces chauffés avec variation de l'humidité et de la température, degrés élevés de pollution comprenant des polluants spécifiques, par exemple locaux techniques électriques dans les usines » ou à des «Espaces non chauffés à humidité relative élevée (jusqu'à $70 \%$ ) avec certains risques de condensation, degrés moyens de pollution, effets possibles de polluants spécifiques, par exemple églises dans des zones polluées, cabines téléphoniques extérieures dans des zones polluées».

- IC5 : corrosivité très forte correspondant à des "Espaces chauffés avec influence de l'humidité relative, degrés élevés de pollution comprenant des polluants spécifiques tels que le $\mathrm{H}_{2} \mathrm{~S}$, par exemple locaux techniques électriques, locaux de raccordement dans les industries sans contrôle efficace de la pollution » ou à des "Espaces non chauffés à humidité relative élevée et risque de condensation, degrés moyens et élevés de pollution, par exemple salles de stockage en sous-sol dans des zones polluées». 
Ce travail a donc pour objectif de comparer des environnements entre eux et de les évaluer de façon absolue grâce à une norme pour une exposition d'un an ; pour des durées plus courtes (transports, travaux, expositions) les résultats peuvent être interprétés avec des lignes directrices rédigées pour un mois ; dans ce cas le taux de corrosion et le classement sont seulement indicatifs (6).

\begin{tabular}{|lccccc|}
\hline Corrosivité & Très pur & Pur & Propre & Peu contaminé & Pollué \\
$\mathrm{nm} /$ mois & $\leq 9$ & $\leq 15$ & $\leq 25$ & $\leq 35$ & $>35$ \\
\hline
\end{tabular}

Classification de la corrosivité du cuivre en atmosphères intérieures pour une durée d'exposition d'un mois d'après Sacchi \& Muller

\section{Conditions expérimentales de mesures}

Vingt-huit vitrines, treize salles, vingt-cinq réserves, l'intérieur de la Porte de l'Enfer au musée Rodin, six caisses ont été étudiées, soit cinquante-neuf espaces permanents et quatorze temporaires (transports, chantiers et expositions). Les quatre cinquièmes des locaux étaient chauffés, soit quatre espaces permanents sur cinq. Les trois quarts des espaces temporaires n'étaient pas chauffés. Deux modèles d'appareils ont été utilisés :

- les capteurs TF50 modèle 610 de la société Rohrback Cosasco de 2002 à 2007 ; ces capteurs très minces $(25 \mathrm{~nm})$ sont bien adaptés aux environnements intérieurs faiblement corrosifs ; leur résolution est de $0,1 \%$ et la reproductibilité des mesures de $\pm 0,5 \%$. Chaque capteur a été testé et étiqueté individuellement, en reportant son épaisseur et sa précision
$( \pm 10 \%)$. Les mesures ont été faites avec un instrument de type CK-4 ${ }^{\circledR}$ Corrosometer, ce qui obligeait à se déplacer pour chaque mesure, d'où une période d'échantillonnage d'un mois ;

- des enregistreurs programmables AirCorr développés par la société nke grâce à un projet de recherche coopérative du sixième programme-cadre ont été utilisés de 2006 à 2011. Les capteurs de ces appareils mesuraient $250 \mathrm{~nm}$ d'épaisseur pour une résolution de 0,1 à $0,2 \mathrm{~nm}$. Ces appareils autonomes permettent de faire une acquisition toutes les heures (7) (8).

Dans les deux cas des capteurs en cuivre ont été choisis pour leur sensibilité aux variations d'humidité et aux polluants intérieurs tels que les acides et la plupart des molécules et radicaux organiques, avec une influence d' $\mathrm{H}_{2} \mathrm{~S}$ et un effet synergique de $\mathrm{SO}_{2}, \mathrm{NO}_{2}$ et de l'ozone $\left(\mathrm{O}_{3}\right)$. Les mesures n'ont pu être dupliquées pour des considérations d'ordre budgétaire (voir tableau page suivante).

Le climat a été documenté avec des enregistreurs Madgetech PRH Temp RH101 (résolution de $0,1{ }^{\circ} \mathrm{C}$ en température, précision étalonnée de $\pm 0,5^{\circ} \mathrm{C}$ de 0 à $50{ }^{\circ} \mathrm{C}$; résolution d'humidité de $0,5 \% \mathrm{HR}$ de 0 à $95 \%$ HR et précision de $\pm 3,0 \% \mathrm{HR}$ ).

\section{Résultats}

Dans $80 \%$ des espaces, la corrosivité est très faible ou faible, soit dans la presque totalité des vitrines, les trois quarts des réserves et la moitié des salles; ces espaces bien adaptés à la conservation des collections patrimoniales ne sont pas obligatoirement

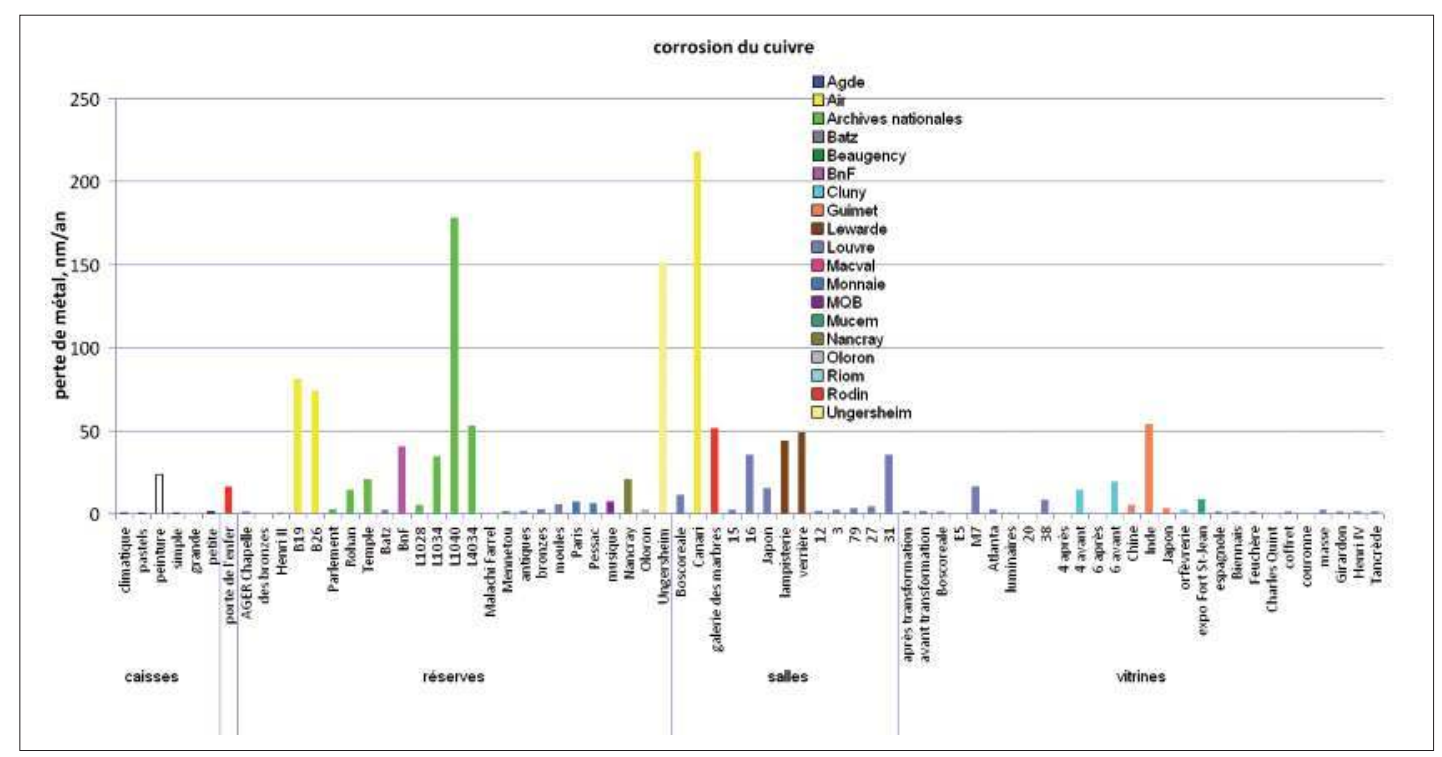

Classement des espaces obtenu par mesurage de la perte de métal estimée en nm/an, d'après ISO $11844-1$ 


\begin{tabular}{|c|c|c|c|c|c|}
\hline Musée & Local & Désignation & Jours & $\mathrm{nm} / \mathrm{an}$ & 11844 \\
\hline de l'éphèbe & vitrine & vitrine après transformation & 389 & 1 & $\mathrm{ICl}$ \\
\hline de l'éphèbe & vitrine & vitrine avant transformation & 78 & 1 & $\mathrm{ICl}$ \\
\hline Louvre & réserve & réserve des bronzes & 427 & 0 & $\mathrm{ICl}$ \\
\hline Louvre & réserve & réserve Henri II & 427 & 0 & $\mathrm{ICl}$ \\
\hline Louvre AGER & réserve & réserve AGER Chapelle & 111 & 1 & $\mathrm{ICl}$ \\
\hline Louvre & salle & salle Boscoreale & 175 & 11 & IC2 \\
\hline Louvre & vitrine & vitrine Boscoreale & 392 & 1 & $\mathrm{ICl}$ \\
\hline Louvre & vitrine & vitrine $\mathrm{E} 5$ & 427 & 0 & $\mathrm{ICl}$ \\
\hline Louvre & vitrine & vitrine $M 7$ & 368 & 16 & $\mathrm{IC} 2$ \\
\hline de l'Air et de l'Espace & réserve & réserve B19 & 367 & 81 & IC3 \\
\hline de l'Air et de l'Espace & réserve & réserve B26 & 367 & 74 & IC3 \\
\hline de l'Air et de l'Espace & salle & salle Canari & 384 & 218 & IC4 \\
\hline Archives nationales & réserve & réserve Parlement & 256 & 2 & $\mathrm{ICl}$ \\
\hline Archives nationales & réserve & réserve Rohan & 256 & 14 & $\mathrm{IC} 2$ \\
\hline Archives nationales & réserve & réserve Temple & 256 & 20 & $\mathrm{IC} 2$ \\
\hline Louvre & vitrine & vitrine expo Atlanta & 220 & 3 & $\mathrm{ICl}$ \\
\hline des marais salants & réserve & réserve Batz & 229 & 2 & $\mathrm{ICl}$ \\
\hline Rodin & extérieur & porte de l'enfer & 151 & 16 & $\mathrm{IC} 2$ \\
\hline Rodin & salle & salle 15 & 151 & 2 & $\mathrm{ICl}$ \\
\hline Rodin & salle & galerie des marbres & 151 & 52 & IC3 \\
\hline BnF site F Mitterrand & réserve & réserve $\mathrm{L} 1040$ & 408 & 178 & IC4 \\
\hline BnF site F Mitterrand & réserve & réserve L1028 & 408 & 5 & $\mathrm{ICl}$ \\
\hline BnF site F Mitterrand & réserve & réserve $\mathrm{BnF}$ & 154 & 40 & $\mathrm{IC} 3$ \\
\hline BnF site F Mitterrand & réserve & réserve L1034 & 408 & 34 & IC3 \\
\hline BnF site $\mathrm{F}$ Mitterrand & réserve & réserve L4034 & 408 & 53 & IC3 \\
\hline Louvre AGER & réserve & petite caisse & 223 & 1 & $\mathrm{ICl}$ \\
\hline Louvre AGER & réserve & grande caisse & 190 & 0 & $\mathrm{ICl}$ \\
\hline LPArt & transport & caisse climatique & 111 & 0 & $\mathrm{ICl}$ \\
\hline Chenue & transport & caisse pastels & 111 & 0 & $\mathrm{ICl}$ \\
\hline LPArt & transport & caisse simple & 111 & 0 & $\mathrm{ICl}$ \\
\hline Louvre peintures & transport & caisse peinture expo Turin & 14 & 24 & IC3 \\
\hline Cluny & salle & salle 16 & 347 & 35 & IC3 \\
\hline Cluny & vitrine & vitrine 20 & 352 & 0 & $\mathrm{ICl}$ \\
\hline Cluny & vitrine & vitrine 4 après & 480 & 0 & $\mathrm{ICl}$ \\
\hline Cluny & vitrine & vitrine 6 après & 480 & 0 & $\mathrm{ICl}$ \\
\hline Cluny & vitrine & vitrine 38 & 358 & 8 & $\mathrm{IC} 2$ \\
\hline Cluny & vitrine & vitrine 4 avant & 347 & 14 & $\mathrm{IC} 2$ \\
\hline Cluny & vitrine & vitrine 6 avant & 347 & 19 & $\mathrm{IC} 2$ \\
\hline Guimet & vitrine & vitrine Chine & 456 & 5 & $\mathrm{ICl}$ \\
\hline Guimet & vitrine & vitrine Japon & 456 & 3 & $\mathrm{ICl}$ \\
\hline Guimet & vitrine & vitrine Inde & 456 & 54 & IC3 \\
\hline Louvre & salle & tapisserie expo Japon & 264 & 15 & $\mathrm{IC} 2$ \\
\hline Centre historique minier & salle & salle lampisterie & 169 & 44 & IC3 \\
\hline Centre historique minier & salle & salle verrière & 169 & 49 & IC3 \\
\hline Macval & réserve & réserve Malachi Farrel & 31 & 0 & $\mathrm{ICl}$ \\
\hline Mandet & salle & salle 12 & 471 & 1 & $\mathrm{ICl}$ \\
\hline Mandet & vitrine & vitrine orfèvrerie & 343 & 2 & $\mathrm{ICl}$ \\
\hline musée d'Auvergne & vitrine & vitrine luminaires & 343 & 0 & $\mathrm{ICl}$ \\
\hline Beaugency & réserve & réserve Mennetou & 31 & 1 & $\mathrm{ICl}$ \\
\hline Rodin & réserve & réserve antiques & 128 & 2 & $\mathrm{ICl}$ \\
\hline Rodin & réserve & réserve bronzes & 128 & 3 & $\mathrm{ICl}$ \\
\hline Rodin & réserve & réserve moules & 128 & 5 & $\mathrm{ICl}$ \\
\hline Monnaie de Paris & réserve & réserve Paris & 19 & 7 & IC2 \\
\hline Monnaie de Paris & réserve & réserve Pessac & 249 & 6 & $\mathrm{IC} 2$ \\
\hline MQB & réserve & réserve musique & 325 & 7 & $\mathrm{IC} 2$ \\
\hline Mucem & vitrine & vitrine expo Fort St-Jean & 168 & 9 & $\mathrm{IC} 2$ \\
\hline des maisons comtoises & réserve & réserve Nancray & 116 & 21 & $\mathrm{IC} 2$ \\
\hline Louvre & salle & salle 3 & 370 & 2 & $\mathrm{ICl}$ \\
\hline Louvre & vitrine & vitrine espagnole & 385 & 1 & $\mathrm{ICl}$ \\
\hline Louvre & salle & salle 79 & 378 & 3 & $\mathrm{ICl}$ \\
\hline Louvre & vitrine & vitrine Biennais & 378 & 1 & $\mathrm{ICl}$ \\
\hline Louvre & vitrine & vitrine Feuchère & 378 & 1 & $\mathrm{ICl}$ \\
\hline Louvre & salle & salle 27 & 358 & 4 & $\mathrm{ICl}$ \\
\hline Louvre & vitrine & vitrine Charles Quint & 169 & 0 & $\mathrm{ICl}$ \\
\hline Louvre & vitrine & vitrine coffret & 344 & 1 & $\mathrm{ICl}$ \\
\hline Louvre & vitrine & vitrine couronne & 344 & 0 & $\mathrm{ICl}$ \\
\hline Louvre & vitrine & vitrine masse & 344 & 2 & $\mathrm{ICl}$ \\
\hline musée béarnais & réserve & réserve Oloron & 60 & 3 & $\mathrm{ICl}$ \\
\hline Louvre & salle & salle 31 & 266 & 35 & IC3 \\
\hline Louvre & vitrine & vitrine Henri IV & 364 & 1 & $\mathrm{ICl}$ \\
\hline Louvre & vitrine & vitrine Tancrède & 378 & 1 & $\mathrm{ICl}$ \\
\hline Louvre & vitrine & vitrine expo Girardon & 126 & 1 & $\mathrm{ICl}$ \\
\hline écomusée d'Alsace & réserve & réserve Ungersheim & 140 & 151 & IC4 \\
\hline
\end{tabular}

Localisation des capteurs, durée d'exposition, classes de corrosion 
chauffés : musée Mandet à Riom, Archives nationales (site de Paris), écomusée des Maisons comtoises à Nancray, réserve du musée de la Monnaie de Paris à Pessac, réserve du musée Béarnais à Oloron, Porte de l'Enfer du musée Rodin ; dans un tiers de ces espaces non chauffés, la corrosivité est moyenne : galerie des marbres du musée Rodin, Centre historique minier à Lewarde, réserves B19 et B26 du musée de l'Air et de l'Espace ; elle est forte dans la halle à chlorures d'Ungersheim.

Inversement la corrosivité peut être importante dans des espaces chauffés, comme le magasin L1040 de la Bibliothèque nationale de France (site François Mitterrand) où la corrosion des armoire climatiques s'explique par les émanations acides des collections et leurs conditionnements dans des magasins confinés, d'où des concentrations dépassant la limite tolérée par la norme ISO 11799 (9) ; l'air n'est pas traité dans la salle 31 du Louvre qui est en relation directe avec l'entrée du musée sous la pyramide ; enfin l'avion Bernard 191 GR n² Oiseau Canari du musée de l'Air et de l'Espace est construit en panneaux de bois qui sont peut-être à l'origine d'une forte corrosion.

Il n'est pas toujours possible d'expliquer la corrosivité d'un environnement : la vitrine Inde du musée Guimet n'est pas sensée émettre de composés acides puisqu'elle est en pierre, verre et métal, et le climat y est correct (température moyenne de mai à novembre de $22 \pm 0,6^{\circ} \mathrm{C}$, humidité relative moyenne de $51 \%$, comprise entre 32 et $58 \%$ ) ; pourtant elle est plus corrosive que les deux autre vitrines du même musée.

Cette méthode permet de vérifier si un changement a été bénéfique : le musée d'Agde a profité de la restauration de l'Ephèbe en 2010 pour remplacer l'éclairage basse tension de la vitrine par des diodes électroluminescentes et changer le socle pour améliorer l'équilibre de la statue. Ces changements modifiant la circulation d'air entre le bac à matériau tampon et le volume de la vitrine, on a vérifié qu'elle restait très faiblement corrosive après sa modification. Au musée de Cluny, les vitrines en chêne et contreplaqué s'avèrent moins corrosives après avoir recouvert le bois d'un pare-vapeur et remplacé le contreplaqué par un composite (constitué d'un noyau en polyéthylène basse densité (LDPE) entre deux feuilles d'aluminium) (10).

La corrosivité est très faible dans plus de la moitié des espaces destinés à conserver des collections sur le long terme, faible et moyenne dans $20 \%$ de ces espaces. Les trois quarts des espaces temporaires

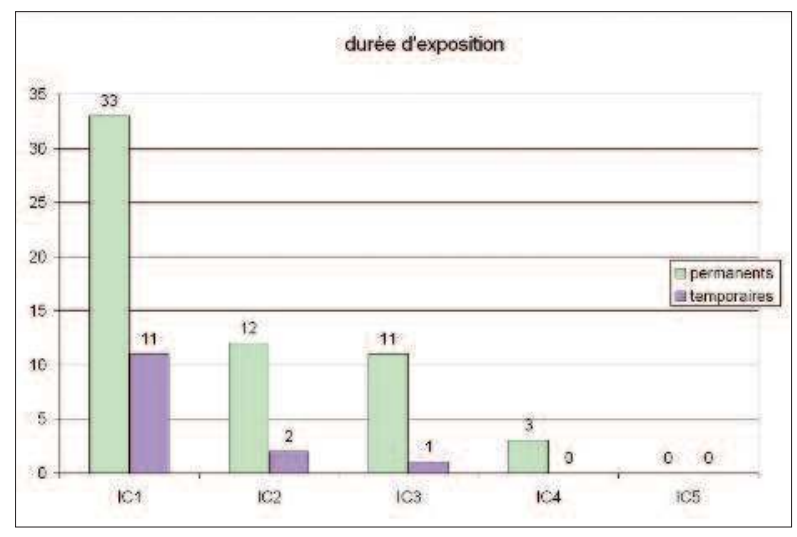

Nombre d'environnements permanents et temporaires classés d'après ISO 11844-1

sont très faiblement corrosifs, $13 \%$ sont faiblement corrosifs, $5 \%$ très fortement corrosifs.

$40 \%$ des salles et $20 \%$ des réserves sont moyennement corrosives, $8 \%$ des réserves et des salles sont fortement corrosives. Les réserves représentent presque la moitié des environnements faiblement corrosifs, les vitrines $38 \%$, les salles $15 \%$. Les réserves et les salles représentent respectivement $42 \%$ des environnements corrosifs, les vitrines et les caisses de transport $8 \%$. Les réserves représentent la moitié des environnements fortement corrosifs, les salles et l'extérieur respectivement un quart.

Quelle que soit la durée d'exposition, le classement IC 1 correspond à celui proposé par Sacchi \& Muller, le classement IC2 de la norme est en bonne corrélation avec la classe $\mathrm{C} 2$ pour quatre études, sousévalué pour huit études pour lesquelles la durée d'exposition variait de 19 à 358 jours, surévalué pour deux études d'un an ; le classement IC3 est sous évalué par Sacchi \& Muller pour l'étude de quatorze jours, en bonne corrélation pour les études de cinq mois, surestimé pour les études d'un an.

\begin{tabular}{|rrrrrrr|}
\hline & IC1 & IC2 & IC3 & IC4 & IC5 & total \\
$\mathrm{C} 1$ & $\mathbf{4 4}$ & 8 & 1 & 0 & 0 & 53 \\
$\mathrm{C} 2$ & 0 & $\mathbf{4}$ & 0 & 0 & 0 & 4 \\
$\mathrm{C} 3$ & 0 & 2 & 4 & 0 & 0 & 6 \\
$\mathrm{C} 4$ & 0 & 0 & 2 & 0 & 0 & 2 \\
$\mathrm{C} 5$ & 0 & 0 & 5 & 3 & 0 & 8 \\
total & 44 & 14 & 12 & 3 & 0 & 73 \\
\hline
\end{tabular}

Croisement des classements de la norme $11844-4$ avec les lignes directrices de Sacchi \& Muller 


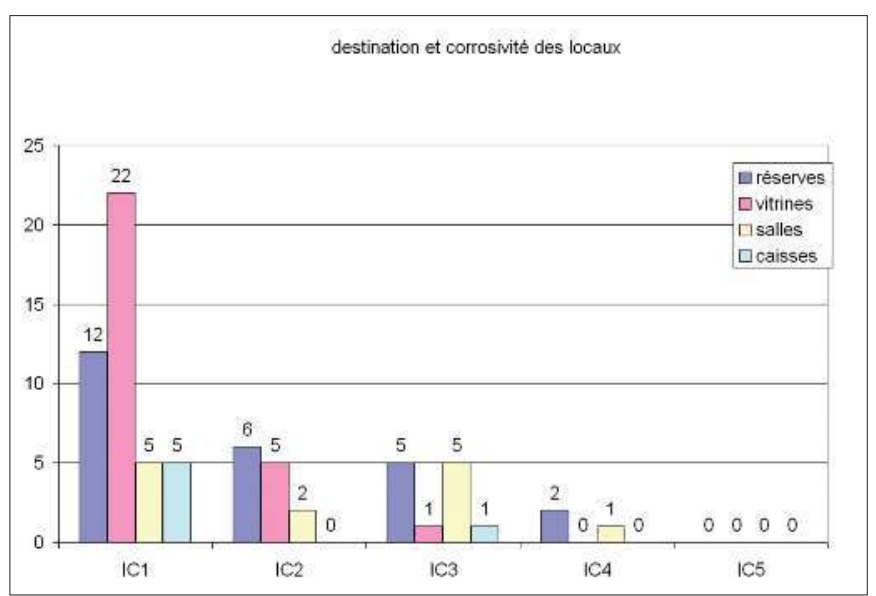

Corrosivité des locaux en fonction de leur utilisation, d'après ISO $11844-1$

\section{Conclusions}

Les méthodes développées dans l'industrie pour évaluer la qualité globale d'environnements faiblement corrosifs en mesurant la vitesse de corrosion d'un métal, donnent des informations quantitatives sans avoir besoin d'effectuer d'analyses coûteuses ; elles trouvent des applications concrètes dans le domaine du patrimoine et permettent de pallier l'absence de normes pratiques pour répondre à des questions simples : les conditions de conservation sont-elles correctes dans ce musée, cette vitrine, ce magasin ? Est-il nécessaire de modifier ces conditions, est-il possible d'améliorer la conservation des collections? Et pendant les transports?

Ces résultats servent de référence pour évaluer des espaces de conservation, comparer entre eux des bâtiments, des vitrines, des réserves, assurer la traçabilité des collections dans le court ou long terme. Ils permettent de répondre aux questions que se posent les responsables de collections françaises en donnant des informations quantitatives, mais également du fait du nombre d'établissements étudiés, de mieux se positionner de façon relative.

Dans le cas d'études supérieures à un an, la norme 11844-1 permet de classer ces espaces en tenant compte des variations climatiques saisonnières et des épisodes de pollution. Il n'existe pas encore de référentiel adapté aux environnements faiblement corrosifs sur le court terme, mais les recommandations de Sacchi \& Muller pourraient être utilisées pour les durées d'exposition d'un mois.
Les enregistreurs de corrosion autonomes développés par la société $n k e$ dans un projet précédent financé par le FP6, sont en cours d'amélioration dans le cadre du projet Musecorr financé par le FP7 (contrat $\mathrm{n}^{\circ}$ : 226 539) ; les capteurs seront facilement interchangeables, les données faciles à récupérer, la transmission automatique des données par e-mail sera possible. Des capteurs de compositions plus proches des collections (argent, plomb, zinc, acier, laiton) ont été développés, avec une sensibilité accrue, permettant de nouvelles plages de mesures, avec une interface de communication universelle, une étanchéité améliorée pour l'exposition en extérieur. L'ergonomie du logiciel a été améliorée pour simplifier l'interprétation des données, en particulier en se référant à des normes ou des recommandations.

\section{Notes}

(1) Fiaud, C. La corrosion atmosphérique, in Corrosion et protection des métaux, Centre français de la corrosion et ministère de la Recherche et de l'Industrie, Les Houches, 26 septembre-4 octobre 1981, pp. 306-315. (2) Leygraf, C. et Graedel, T. Atmospheric corrosion, The Electrochemical Society Inc., Pennington : Wiley-interscience, 2000, 354 p.

(3) Observatoire de la qualité de l'air intérieur www.oqai.fr/obsairint.aspx, consulté le 10 janvier 2012

(4) Prosek, T., Kouril, M., Hilbert, L. R., Degres, Y., Blazek, V., Thierry, D. et Hansen, M.- $\varnothing$. Real time corrosion monitoring in the atmosphere using automated battery driven corrosion loggers, Corrosion Engineering, Science and Technology n43, 2008, pp. 129-133.

(5) ISO/DIS 1 1844-1 : Corrosion of metals and alloys - classification of low corrosivity of indoor atmospheres - Part 1 : determination and estimation of indoor corrosivity, ISO, 2006, 18 p.

(6) Sacchi, E. et Muller, C. Air quality monitoring at historic sites - redefining an environmental classification system for gaseous pollution. Atlanta GA : American Society of Heating, Refrigerating \& Air-Conditioning Engineers, Inc., USA, 2005, 7 p

(7) Collectif : Automated corrosion sensors as on-line real time process control tools (CORRLOG), Co-operative Research Project, Sixth Framework Programme, Horizontal Research Activities Involving SMEs, CoOperative Research, Contract no. 018207, 09/2005-02/2008, 2008.

(8) Prosek, T., Kouril, M., Dubois, F., Scheffel, B., Dubus, M., Degres, Y., Hubert, V., Taube, M. et Thierry, D. Corrosion monitoring in model indoor atmospheres polluted with organic acids using automated real time corrosion sensors, European Workshop and Training Day on Cultural Heritage Preservation, Berlin, Germany, September 26 to 282011.

(9) ISO 11799 Information and documentation. Document storage requirements for archive and library materials, 2004, 15 p.

(10) Dubus, M. et Laurent, A.-M. Tout ce que vous devez savoir sur les vitrines en bois, Technè n²9, 2009, pp. 101-108. 